\title{
Cancer Metastases Prevention by Photodynamic In-vivo Detection and Destruction of Circulating Tumor Cell Clusters
}

\author{
Detlef Schikora $^{1, ~ *}$, Michael Weber ${ }^{2}$ \\ ${ }^{1}$ Department of Biophysics, Faculty of Science, University of Paderborn, Paderborn, Germany \\ ${ }^{2}$ Laser Clinic Lauenfoerde, Cancer Division, Lauenfoerde, Germany \\ Email address: \\ detlef.schikora@uni-paderborn.de (D. Schikora), praxisdrmweber@gmx.com (M. Weber) \\ ${ }^{*}$ Corresponding author
}

To cite this article:

Detlef Schikora, Michael Weber. Cancer Metastases Prevention by Photodynamic In-vivo Detection and Destruction of Circulating Tumor Cell Clusters. International Journal of Clinical Oncology and Cancer Research. Vol. 4, No. 5, 2019, pp. 38-44.

doi: 10.11648/j.ijcocr.20190405.11

Received: October 8, 2019; Accepted: November 6, 2019; Published: November 13, 2019

\begin{abstract}
We demonstrate a new diagnostic method, the Photodynamic Infrared Spectroscoppy (PDIS), which is able to detect circulating tumor clusters and circulating tumor cells in the circulatory system. In particular the circulating tumor clusters are agreed as the main cause for metastases formation and therefore for the cancer mortality. The cancer mortality rate is unchanged worldwide in the last twenty years, indicating the absence of clinically effective metastases prevention therapies. No diagnostic method is existing, which allows to detect tumor clusters in vivo in the blood, which is the prerequisite for tumor cluster destruction and metastases prevention. The PDIS method is based on photodynamic physics and high resolution spectroscopy and is using calibrated spectroscopic data for the diagnostic analysis of the blood screening spectra. In the paper we are focussing to brest cancer diagnostics using the photosensitizer Indocyangreen. A flow appartus is described, which enables to calibrate the fluorescence spectra of single tumor cells and single tumor clusters. Due to the calibrated diagnostic data, the PDIS enables the identification of circulating tumot cells and tumor clusters in the bloodstream with the ultimate accuracy of one cluster per 61 blood and with a sensitivity of $98 \%$. Circulating tumor cell clusters are formed only in solid tumors, that's why they are appropriate objects to validate cancer treatments and to recognice cancer formation. Due to the one-to one correspondence between tumor clusters in blood and the existence of solid tumors in the organism, the PDIS as diagnostic method can be used to control and to verify any oncologic treatment with respect to its clinical efficay. The common "wait and hope" strategy after finishing a chemotheraypy can be replaced by an effective follow-up strategy. Furthermore, the PDIS diagnostics can be used to recognize a tumor formation in the earliest possible stage, the carcinom in-situ stage. Finally the in-vivo detection of tumor clusters in the blood enables the immediate and controlled destruction of the these clusters by Photodynamic Therapy or other oncologic methods and therefore an increase of the overall survival of cancer patients and a decrease of the cancer mortalitay rate.
\end{abstract}

Keywords: Photodynamic Diagnosis, Circulating Tumor Cell Clusters, Fluorescence Spectroscopy, High Resolution Blood Screening, Cancer Metastases Prevention, Cancer Treatments Verification

\section{Introduction}

Metastases are the main cause of cancer-related deaths, however, the mechanisms of metastatic spread are not completely understood. It is a complicated, multistep process that requires detachment of cancer cells from the primary tumor, their intravasation into the bloodstream, survival of CTC and CTCL in the bloodstream and finally extravasation to distant organs [1]. Besides the circulating tumor cells, the tumor clusters seem to play a key role in the metastatic process [2]. In 1954, Watanabe showed that these clustered cells have high metastatic potential [3]. More recent studies indicated that clusters have distinct features compared to single cells, including their phenotype, the gene expression signature and the dissemination mode. Thus, establishing the role and significance of circulating tumor clusters in cancer 
spread is of extremely importance. Circulating tumor clusters are defined as a group of more than two or three tumor cells with strong cell-cell-contacts. They are rare, but highly metastatic. Despite representing only $2-5 \%$ of all circulating tumor cells, clusters were shown to form about $50 \%$ of breast cancer metastases and their metastatic potential was estimated to be 23-50 times higher than that of single cells [4]. Tumor clusters represent a conglomerate of tumor cells and other types of cells including platelets, immune cells and cancer-associated fibroblasts. It seems that tumor clusters are epithelial-mesenchymal hybrids and therefore of enormous plasticity [5]. This composition provides a local microenvironment that is thought to protect tumor clusters from death in the circulation by minimising shear stress, immune attack and through facilitating colonisation [6]. Au et. al. have shown that clusters can actually traverse capillary-sized vessels [7]. It appears that tumor clusters, upon constriction, undergo rapid reorganization, forming a chain-like structure, which reduces hydrodynamic resistance and allows them to pass through a small vessel. This reorganization was shown to be reversible, since after exiting constriction, clusters reform into their typical organisations. Aceto et al. [4] have demonstrated that the invasive potential of tumor clusters is higher than of single tumor cells. Cheung et al. obtained similar results and estimated that as much as $97 \%$ of metastases arise from clusters [8]. Other data suggested that intravascular aggregation/proliferation could be excluded as potential source of tumor clusters due to the unfavourable conditions present in the bloodstream [9]. Very recently, Gkountela et. al. [10] demonstrated that- in strong contrast to circulating tumor cells- the transcription factors pattern of tumor clusters reveal similarity to embryonic stem cells and increased proliferation.

Tumor clusters are of particular diagnostic importance because they only occur, when a primary tumor exists. Tumor clusters thus may serve as indicators for the formation and existence of primary tumors and may therefore - vice versa also serve as indicators for a complete or non-complete removal of primary tumors [4]. Cancer metastases arise mainly from circulating tumor cell clusters. Therefore, they should be the most important targets for metastases prevention and reduction [8]. The existing liquid biopsy methods of circulating tumor cell detection, like Cell Search [12] cannot be applied as reference for tumor clusters enumeration, because they do not preserve their morphological and molecular status and they are sensitive only for epithelial cells. There is a lack of a gold-standard method of detecting tumor clusters, which enables a comparison of the results obtained by different methods. Therefore, there is a great need for a standardised method for isolating and for detecting circulating tumor clusters [2]. The PDIS is based on photodynamic physics, which was discovered and described for the first time by A: Jablonski already in 1933 [11]. Photodynamic excitation is a physical principle, which is explained in the Jablonski-Diagram. The most important characteristic feature of a photodynamic process is the simultaneous emission of fluorescence and phosphorescence radiation, if a photosensitive substance is excited by light. This is a kind of „fingerprint“" and proves doubtless the photodynamic origin of the signals. Therefore, in spectroscopic peak analyses, photon peaks generated by the environment can be clearly distinguished from photon peaks generated by a photodynamic process. In this paper, we have used indocyanine green (ICG) as specific liposomal formulations, which have an absorption wavelength of 785 $\mathrm{nm}$, a fluorescence wavelength of $830 \mathrm{~nm}$ and a phosphorescence wavelength of $940 \mathrm{~nm}$.

\section{Materials and Methods}

Methods for in vivo detection of circulating tumor clusters and circulating tumor cells do not exist. Due to the lack of evidence based reference procedures and standards regarding in-vivo tumor cluster detection, we have calibrated the PDIS.

Figure 1 shows a scheme of the flow calibration apparatus.

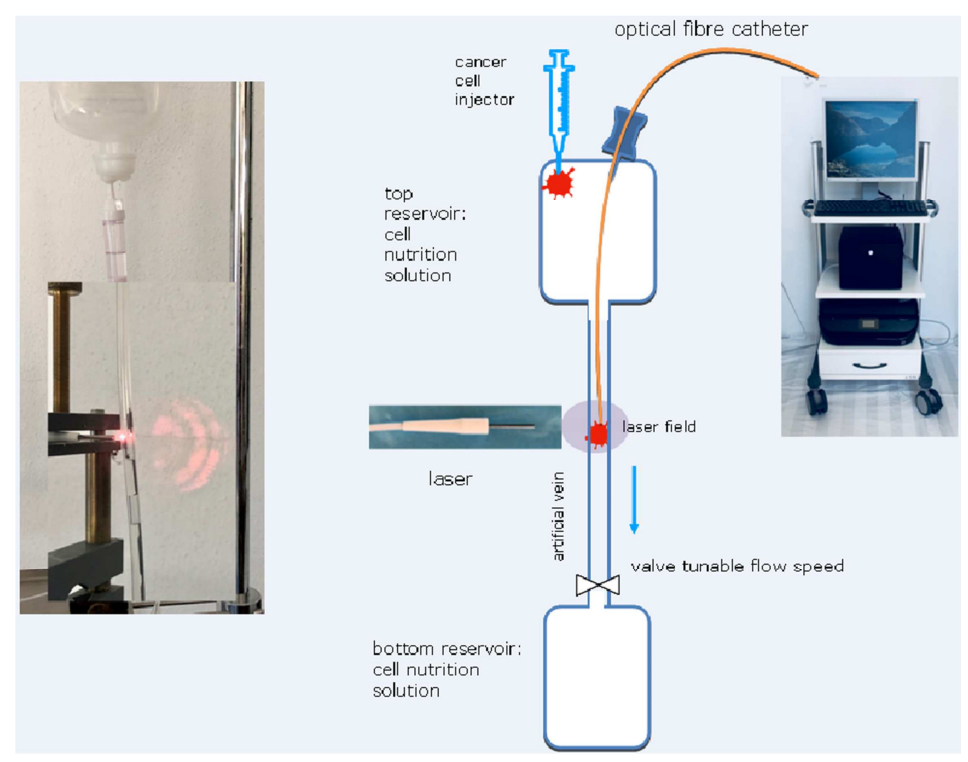

Figure 1. Flow calibration apparatus for circulating tumor clusters and cells. 
An optical fibre detector is placed within a flexible $4 \mathrm{~mm}$ in diameter and $40 \mathrm{~cm}$ long silicone tube, representing a mid-. sized vein. The distal end of the optical fibre detector is coupled to the high resolution-spectrometer. The whole assembly is placed in a clean-room box, maintained for measurements at a const. temperature of $37^{\circ} \mathrm{C}$ and kept under dark conditions during the measurements. The top reservoir of a volume of $500 \mathrm{ml}$ cell nutrition solution contains a port for cancer cell injection and a port for the optical fibre lead through. At the bottom of the top reservoir a flexible silicon tube is arranged, which connects the top and the bottom cell nutrition reservoir. The cell nutrition solution is flowing in the flexible silicon tube from the top to the down reservoir. A valve at the bottom reservoir is used to tune the flow speed, in all experiments the flow speed was kept constant at $10 \mathrm{~cm} / \mathrm{s}$. At the end of the optical fibre detector an external laser source, emitting at $785 \mathrm{~nm}$ and $10 \mathrm{~mW}$ power is arranged, to generate an infrared light field within the silicon tube. When an ICG-incubated tumor cell or tumor cell cluster is flowing through the laser spot, fluorescence radiation at $830 \mathrm{~nm}$ and phosphorescence radiation are excited and guided via optical fibre to the spectrometer. After $4 \mathrm{~h}$ incubation in $15 \mu \mathrm{M}$ ICG solution, the cells, which were plated in a 6-well tissue culture plates at a concentration of 10 cells/well were injected into the top nutrition reservoir. The ICG uptake of MDA 231 and SK-Br-3 cancer cell clusters was studied by hematological investigations using Confocal Scanning Laser Microscopy (CSLM). Confocal Laser Scanning Microscopy (CLSM) images were acquired by a VisiScope (Visitron Systems, Germany). Liposomal ICG was bought from Burg-Apotheke Königstein, the spectroscopic device was produced by Hamamatsu Ltd, Japan. The recorded spectra were statistically analysed with respect to peak emission wavelength (nm), peak FWHM-broadness (s), peak intensity (counts) and peak origin (simultaneous fluorescence and phosphorescence emission).

\section{Results}

\subsection{ICG Uptake}

Figure 2 illustrates the uptake of liposomal ICG after $4 \mathrm{~h}$ in $15 \mu \mathrm{g} / \mathrm{ml}$ solution concentration, measured by Confocal Laser Scanning Microscopy (CLSM). The liposomal ICG was accumulated inside the SK Br 3 breast cancer cluster (top), consisting of 3 cells and also in SK Br 3 cells (middle).

The ICG is marked by the red color, the cell nuclei were not affected. In contrast, the monozytes/ leucozytes did not accumulate ICG at same concentrations of about $15 \mu \mathrm{g} / \mathrm{ml}$, as shown in the CLSM-picture below.

The infusion concentration of liposomal ICG, which we have used in diagnostic screenings, was always $15 \mu \mathrm{g} / \mathrm{ml}$. At this concentration, which is normally used for diagnostic screenings, the human blood cells, in particular the leucozytes did not show any photosensitizer accumulation [14]. The deciding prerequisites for photodynamic excitation of the breast cancer cells are fulfilled, the circulating breast cancer clusters and cells accumulate the liposomal iCG, which was infused, wheras the leucozytes do not uptake the photosensitizer. Therefore, there are two components in the bloodstream which emit the $830 \mathrm{~nm}$ fluorescence: On the one hand the infused liposomal ICG, which is to $98 \%$ bounded at plasma proteins such as albumin and which forms a constant, continuous fluorescence radiation background. On the other hand, the low number of circulating breast tumor cells and tumor clusters, which have accumulated the liposomal ICG and which supply discrete fluorescence signals upon excitation. That's why it was possible to distinguish unambiguously in the diagnostic spectra the continuous background, which was mathematically compensated to zero intensities and the circulating tumor cells and tumor cluster signals, which appear as discrete fluorescence peaks above the zero intensity background level.
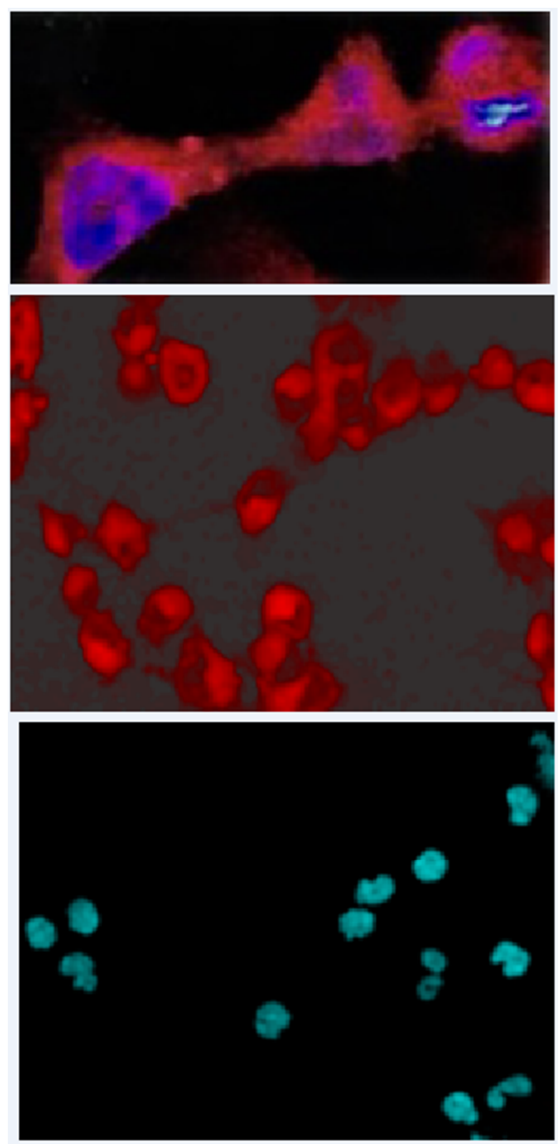

Figure 2. CLSM image showing the ICG uptake of SK Br 3 breast cancer cells (middle) and a Sk-Br 3 cluster incubated for $4 h$ at concentrations of liposomal ICG of about $15 \mu \mathrm{g} / \mathrm{ml}$. All cells were counterstained with DAPI stain. The monozytes do not uptake ICG at this concentration. (below).

\subsection{Calibration Spectra}

\subsubsection{Calibration of Single Tumor Cells}

To get a reference spetrum for the cluster identification, we have determined the fluorescence spectra of single tumor cells. The calibration procedure was performed in a dark environment. During passing the $785 \mathrm{~nm}$ laser spot, the ICG 
incubated cancer cell emits $830 \mathrm{~nm}$ fluorescence and $940 \mathrm{~nm}$ phosphorescence peaks, which were guided to the spectrometer via optical laser catheter. The DMEM nutrition solutions used here were optically inert. The spectroscopic background radiation was compensated by special subtracting software so that the starting intensity was always near the zero fluorescence intensity value. The advantage is that just the photons emitted from the moving cancer cells are displayed above the spectral noise. The flow speed was held constant at $10 \mathrm{~cm} / \mathrm{s}$, which is near the value of the blood flow velocity in peripheral veins. It is obvious from Figure 3 that the $830 \mathrm{~nm}$ fluorescence peak consists of a sharp peak, indicating that the tumor cell has passed the optical window. As long the cell is outside the laser spot, just small intensity fluctuations (statistical noise) are recorded. If the SK-Br 3 cell is directly in front of the optical fibre window, the fluorescence intensity steeply increases to a maximum of about 2000 counts. When the tumor cell is leaving the laser spot, the intensity I $\mathrm{f}$ (d) decreases exponentially, according to $1 / \mathrm{d}^{2}$ (d-distance). The „Full Width at Half Maximum“ (FWHM) of the single peaks is of about $1,2 \mathrm{~s}+/ 0,2 \mathrm{~s}$, in accordance to the flow speed of 10 $\mathrm{cm} / \mathrm{s}$. From a moving object, we have to expect an exponential decay of the fluorescence intensity according to the distance law, if the object is leaving the light field. This is exactly, what the peak in Figure 3 shows. The exponential decay clearly proves the movement of the tumor cell through the laser spot.

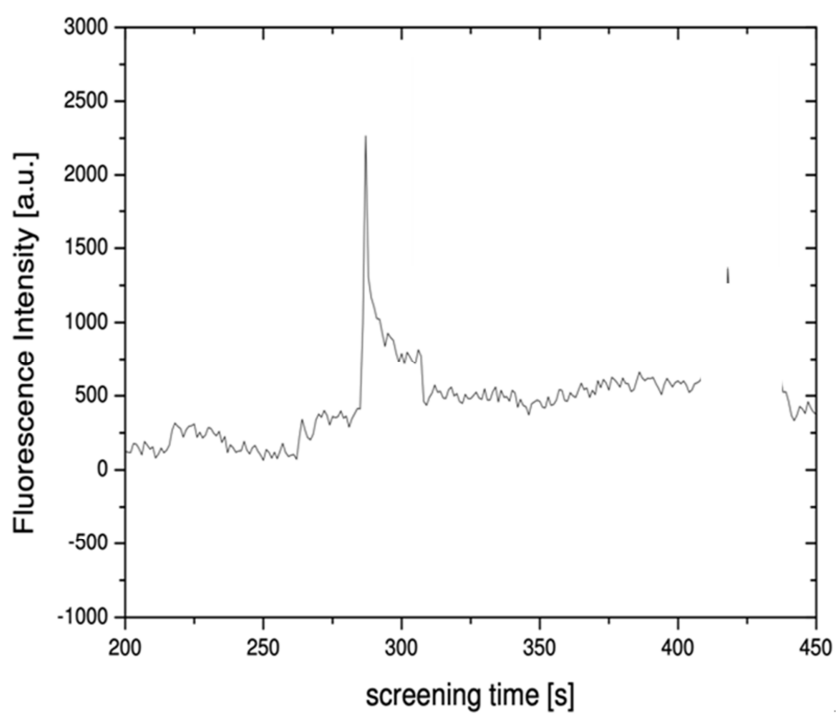

Figure 3. Flow calibration fluorescence spectrum of a single SK-Br 3 tumor cell.

The peak analysis of Figure 3 results in a parameter set for a calibrated single cancer cell of:

emission wavelength: $\lambda_{\mathrm{F}}=830 \mathrm{~nm}, \lambda_{\mathrm{P}}=940 \mathrm{~nm}$

peak broadness: $\mathrm{FWHM}=1,1+/-0,2 \mathrm{~s}$

peak intensity: $\sim 2500$ counts $+/-200$ counts

peak shape: exponential intensity decay

peak origin: $\mathrm{t}\left(\mathrm{Peak}_{\mathrm{F}}\right)=\mathrm{t}\left(\mathrm{Peak}_{\mathrm{P}}\right)=$ photodynamic origin

This peak parameter set of a single moving tumor cell represents the numerical average of 120 experiments.

\subsubsection{Calibration of Single Tumor Clusters}

Figure 4 shows a calibrated fluorescence spectrum of a single SK-Br 3 breast cancer cluster, consisting of three SK-Br 3 cells.

The calibration procedure was performed under the same conditions as described above for the singe tumor cell. It is obvious from Figure 4 that the $830 \mathrm{~nm}$ fluorescence peak consists of three sharp consecutive peaks, indicating that the cluster has passed the optical window as chain of three bounded cells, shown in the inlet of Figure 3. As long the cluster chain is outside the laser spot, just small intensity fluctuations (statistical noise) are recorded. If the SK-Br 3 cluster chain is passing the optical fibre window, the fluorescence intensity steeply increases $3 \mathrm{x}$ within a short time leading to a superposition of three fluorescence maxima. When the cancer cluster is leaving the laser spot, the intensity I f (d) decreases again exponentially. As result, we get a calibrated peak-parameter set for a single moving SK-Br 3 breast cancer cluster:

emission wavelength: $\lambda_{\mathrm{F}}=830 \mathrm{~nm}, \lambda \mathrm{P}=940 \mathrm{~nm}$

peak broadness: FWHM $=3,6 \mathrm{~s}$, which is $3 \mathrm{x}$ the FWHM of a single cell.

peak intensity: $\sim 2000$ counts $+/-200$ counts

peak shape: exponential intensity decay

peak origin: $\mathrm{t}\left(\mathrm{Peak}_{\mathrm{F}}\right)=\mathrm{t}\left(\mathrm{Peak}_{\mathrm{P}}\right)=$ photodynamic origin

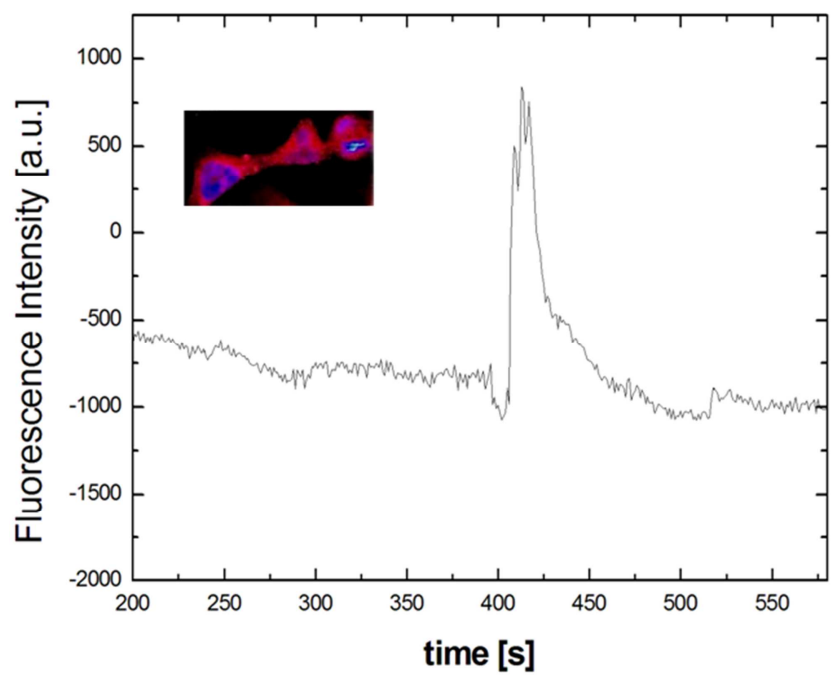

Figure 4. Fluorescence calibration spectrum of a moving 3-cell cluster chain of $\mathrm{SK}-\mathrm{Br} 3$ cell type.

If we compare the calibrated cluster parameter set with the calibrated parameter set of a single cell, we get an accordance in four of the five parameters. Just the peak broadness is different, the cluster peak broadness is $3 \mathrm{x}$ bigger than the FWHM of a single tumor cell. That means in clinical PDIS spectra one can distinguish clearly a tumor cluster from a tumor cell by the parameter FWHM/peak broadness.

The calibrated peaks and peak parameters of a circulating tumor cluster and a circulating tumor cell were used for comparison with clinical peaks measured at breast cancer patients to analyse and to interprete the PDIS blood screening spectra. 


\subsection{Clinical Identification of Tumor Clusters in the Bloodstream}

After infusion of $15 \mathrm{mg}$ liposomal ICG solved in $100 \mathrm{ml}$ $0,9 \% \mathrm{NaCl}$, the optical laser catheter was placed into an arm vein (vena basilica) and the PDIS screening was started.

The extravasal arranged laser tip was placed near above the optical fibre window. In a first step the photodynamic properties of the blood were investigated by activation the 785 nm laser.

The recorded spectrum of Figure 5 shows, besides the narrow laser peak, a clear fluorescence peak at $830 \mathrm{~nm}$ and a phosphorescence peak at $940 \mathrm{~nm}$.

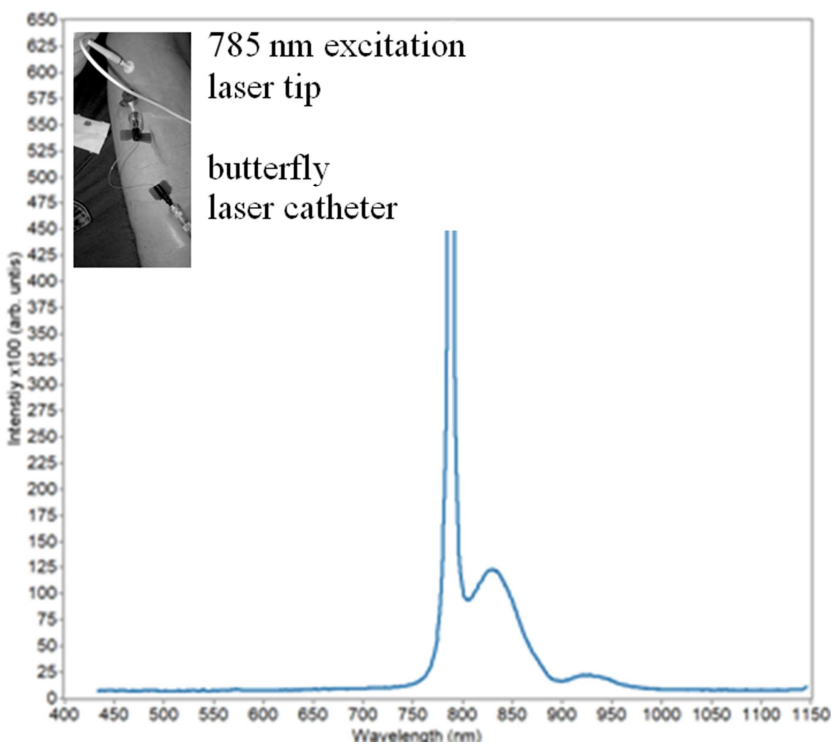

Figure 5. Photodynamic spectrum of the blood after infusion of liposomal $I C G$. The laser excitation power was $20 \mathrm{~mW}$, the excitation wavelength was $785 \mathrm{~nm}$. Besides the narrow laser peak a clear fluorescence peak at $830 \mathrm{~nm}$ and a phosphorescence peak at $940 \mathrm{~nm}$ are shown.

This spectrum confirms that the infused ICG was bounded in the blood to plasmaproteines, in particular to albumin. Therefore, the photodynamic conditions for PDIS screening of circulating tumor clusters and cells are fulfilled. When tumor cells or tumor cell clusters are circulating in the blood, they also will accumulate the liposomal ICG.

After finishing the PDIS blood screening the blood spectrum was measured again, to make sure that the photodynamic conditions were stable over the whole blood screening procedure of about $1000 \mathrm{~s}$.

After confirming the photodynamic prerequisite for PDIS blood screening, the screening can be started as second step. The diagram in Figure 6 shows the PDIS spectrum of a mamma carcinoma patient, 44 years of age, having a ductal, non-metastatic cancer of the left breast, stage 2. The patient got a surgery, the PDIS diagnosis was performed six weeks after.

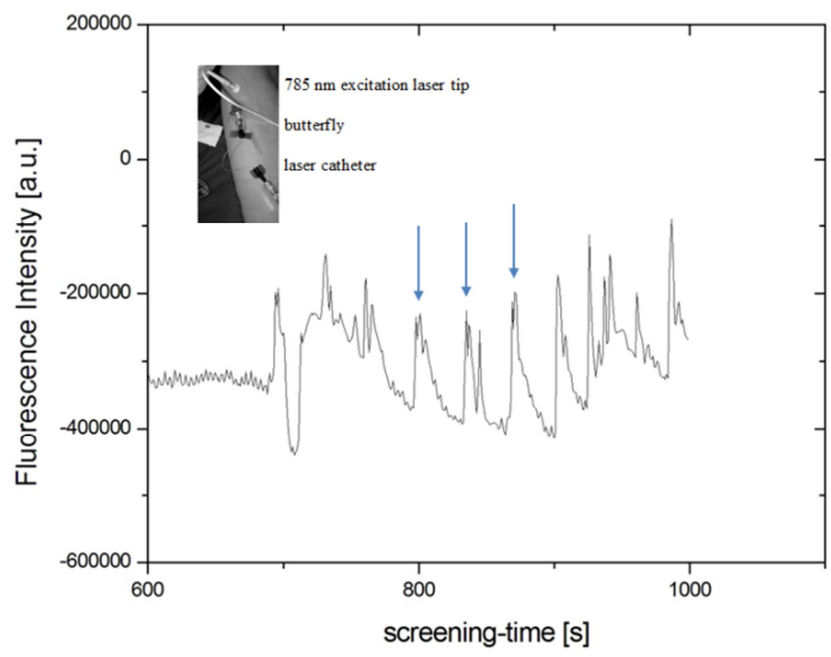

Figure 6. PDIS blood screening spectrum of a mamma carcinoma patient, showing the existence of linear CTC cluster chains in the bloodstream of the patient. The optical fibre detector was placed in the vena basilica, the laser power was $20 \mathrm{~mW}$, and the excitation wavelength was $785 \mathrm{~nm}$.

When we compare the peaks in the clinical spectrum with the calibrated peak for a three-cell cluster chain of Figure 4, the similarity becomes obviouos.

The marked peaks at $800-850 \mathrm{~s}$ screening time show three close consecutive main peaks. The intensity decays exponentially intensity, like the calibrated peak of Figure 4. The fluorescence emission wavelength is $\lambda \mathrm{F}=830 \mathrm{~nm}$, like the calibrated peak, the correspondend phosphorescence peak appears at $940 \mathrm{~nm}$ at the same time, confirming the photodynamic origin of that peak. The peak FWHM is in average of about 3-4 s, which agrees with the calibrated FWHM data. This proves that the marked peaks of Figure 6 were emitted from circulating tumor clusters chains. This result indicates that the surgery was not succesful, the primary tumor was not completely removed. Otherwise no circulating tumor clusters would appear in the bloodstream. The risk of metastases formation is high, the clusters have to be removed immediately from the blood and the remaining tumor tissue must be destroyed.

To destroy the circulating tumor clusters, we have used the photo-oxidative killing of the clusters by infrared laser blood irradiation. The photo-oxidative killing of tumor cells by ICG was reported by Bäumler et. al. [13]. The laser catheter, which was placed in the arm vein for the PDIS diagnostics, was now removed from the spectrometer and coupled to a $785 \mathrm{~nm}$ laser source. The laser blood irradiation (LBI) was performed with $785 \mathrm{~nm}$ infrared laser of $40 \mathrm{~mW}$ power and $30 \mathrm{~J} / \mathrm{cm}^{2}$ dosage. The therapeutic effect of the laser blood irradiation was again controlled by PDIS diagnostics. The PDIS screening after LBI is shown in Figure 7.

The final PDIS spectrum of Figure 7 shows just noise without any peak-signals from circulating tumor cells and tumor clusters. The Photodynamic Therapy was succesful, the clusters were removed and the risk of formation of metastases was reduced. 


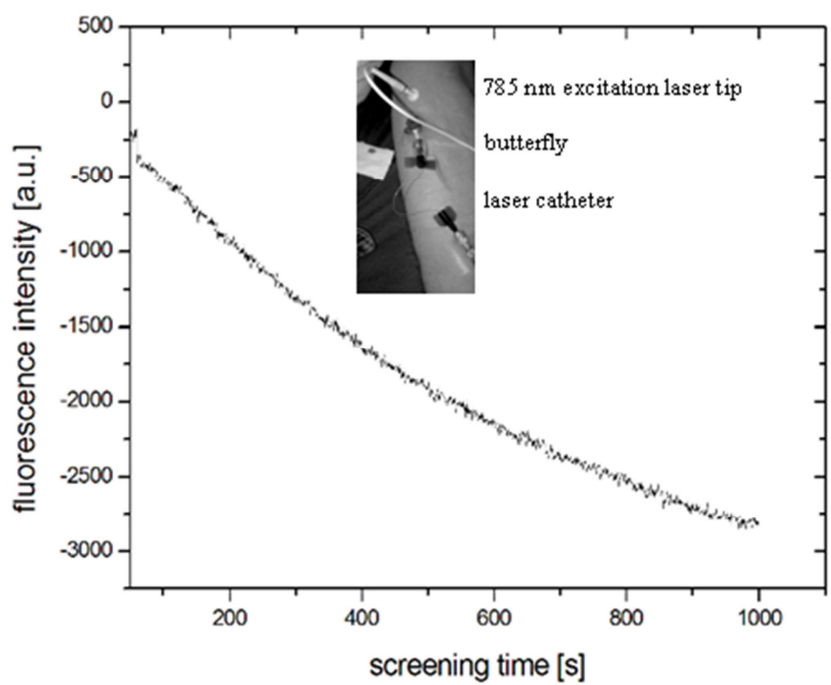

Figure 7. PDIS blood screening spectrum of the mamma carcinoma patient after photo-oxidative killing of the circulating tumor clusters by $785 \mathrm{~nm}$ laser blood irradiation.

\section{Discussion}

The discontinuous emission of CTC clusters reported by Aceto et al 4 is in agreement with the PDIS spectra shown in Figure 6. Obviously, the primary tumor emits the breast cancer clusters discontinuously.

The statistical analysis of 185 patients with non-metastasised breast cancer diagnosis after ICG infusion results in a sensitivity of circulating tumor cluster detection of about 98\%. In this case, "positive" wrong or "negative" wrong results can be practically precluded. When a circulating tumor cell or a circulating tumor cluster is detected in the bloodstream, whose five peak parameters agree with the calibrated breast cancer cell data, the circulating tumor cell or the tumor cell cluster is of breast cancer type. It must be pointed out that PDIS diagnosis cannot be used presently to identify different cancer types of circulating tumor cells clusters, because the quantitative dependence of the amount of ICG, which is accumulated in the circulating tumor cells and clusters of different tumor cell types is not known and must be calibrated in future studies. However, when the type of the solid tumor is known, the primary diagnostic information of PDIS is:

Are there CTC clusters and CTCs in the bloodstream or not?

The PDIS spectra contain precise, calibrated diagnostic information about the presence or the absence of the hazardous tumor clusters in the bloodstream.

Although demonstrated here just for breast cancer, the PDIS diagnostics can be performed for all solid tumor types., provided that flow calibrated data are available. PDIS data allow the therapists to confirm or to change the applied individual therapeutic strategy and to recognise the development of resistance's against the chemotherapeutics.

For healthy persons without tumor, PDIS detects either zero or a very low number of circulating tumor cell peaks, similar to CellSearch [12]. However, we have never detected tumor clusters in the blood of healthy probands. The investigations of Aceto et. al [4] and Cheung et. al [8] have demonstrated that circulating tumor clusters are always formed in tumors and cannot be formed in vessels by aggregation. Therefore, the tumor clusters seem to be appropriate indicators/precursors of the existence of a primary, solid tumor and are-vice versa-appropriate indicators of the complete or not-complete destruction of primary tumors. When tumor cluster appear in the bloodstream after a breast cancer treatment, like surgery or chemotherapy, the primary tumor was not completely removed. PDIS allows to control the dissolution of circulating tumor clusters and to control regularly the blood status regarding tumor clusters. The use of PDIS blood screening after finishing a chemotherapy makes it possible to provide patients with calibration-based informations about their status, thereby reducing psychological stress, fear and uncertainties that are all consequences of the current "Wait and hope" principle.

The liposomal ICG is safe and free of side effects; ICG is used in angiography since many decades. The procedure is easily to perform, the infusion of the liposomal ICG is the only minimal-invasive treatment step. Further flow calibrations of PDIS spectra for different cancer types and different approved photosensitizers like Photofrin are necessary and will be performed in the near future.

\section{Conclusion}

We have developed a new diagnostic method, the Photodynamic Infrared Spectroscoppy (PDIS), which is able to detect circulating tumor clusters and circulating tumor cells in the circulatory system. It is agreed and published in many papers $(2,3,4,5)$ that circulating tumor clusters are agreed the main cause for metastases formation and therefore for the resulting cancer mortality. Despite the enormous research activities, the cancer mortality rate is unchanged worldwide in the last twenty years, indicating the absence of any clinically effective metastases method can close this gap, it allows to detect clearly tumor clusters in-vivo in the bloodstream.

Due to the calibrated diagnostic data, the PDIS enables the identification of circulating tumor cells and tumor clusters in the bloodstream with the ultimate accuracy of one cluster per 61 blood and with a sensitivity of $98 \%$.

Circulating tumor cell clusters are formed only in solid tumors, that's why they are appropriate objects to validate cancer treatments and to recognice cancer formation. Due to the one-to one correspondence between tumor clusters in blood and the existence of solid tumors in the organism, the PDIS as diagnostic method can be used

to control and to verify any oncologic treatment with respect to its clinical efficay. The common "wait and hope" strategy after finishing a chemotheraypy can be replaced by an effective follow-up strategy.

to recognize a tumor formation in the earliest possible stage, the carcinom in-situ stage. A cancer development can be recognized much earlier by PDIS compared to Magneto Resistance Tomography. 
-for in-vivo detection of tumor clusters in the blood, which enables the immediate and controlled destruction of the these clusters by Photodynamic Therapy or other oncologic methods and therefore an increase of the overall survival of cancer patients and a decrease of the cancer mortalitay rate.

The PDIS diagnostics is described here for breast cancer patients, however it can be applied for all types of tumors, except for leucemia. The diagnostic procedure is minimalinvasiv, easy and safe to perform and without any side-effects. The diagnostic result can be obtained within $45 \mathrm{~min}$.

\section{Acknowledgements}

The authors thank the technical support of the University of Paderborn.

\section{Ethical Approval and Consent to Participate}

All PDIS diagnoses on patients were performed after each patient gave his written consent. The diagnostic treatment procedure was approved as NIS according to AMG $\S 40$, MPG $\S$ 20 und der GCP-Verordnung (GCP-V), by Landesärztekammer Niedersachsen. Helsinki Declaration: The authors declare that the diagnostics of the patients were performed under strict consideration of the WAMA most recent (2012-2013) revision of the Declaration of Helsinki (DoH).

\section{Conflicts of Interest}

The authors declare no conflicts of interest.

\section{Funding}

This work was possible by funding of the technical staff by the University of Paderborn.

\section{References}

[1] Massague J, Obenauf AC. Metastatic colonization by circulating tumour cells. Nature. 2016; 529 (7586): 298-306. doi: 10.1038/nature17038.
[2] Anna Fabisiewicz, Ewa Grzybowska CTC clusters in cancer progression and metastasis Med Oncol (2017) 34: 12 DOI 10.1007/s12032-016-0875-0.

[3] Watanabe S. The metastasizability of tumor cells. Cancer. 1954; 7 (2): 215-23.

[4] Aceto N, Bardia A, Miyamoto DT, Donaldson MC, Wittner BS, Spencer JA, et al. Circulating tumor cell clusters are oligoclonal precursors of breast cancer metastasis. Cell. 2014; 158 (5): 1110-064. 22. doi: 10.1016/j.cell.2014.07.013.

[5] Aceto N, Toner M, Maheswaran S, Haber DA. En route to metastasis: circulating tumor cell clusters and epithelial-to-mes- enchymal transition. Trends Cancer. 2015; 1 (1): 44-52. doi: 10.1016/j.trecan.2015.07.006.

[6] Hong Y, Fang F, Zhang Q. Circulating tumor cell clusters: what we know and what we expect (review). Int J Oncol. 2016; doi: 10.3892/ijo.2016.3747.

[7] Au SH, Storey BD, Moore JC, Tang Q, Chen YL, Javaid S, et al. Clusters of circulating tumor cells traverse capillary-sized vessels. Proc Natl Acad Sci USA. 2016; 113 (18): 4947-52. doi: 10.1073/pnas. 1524448113 .

[8] Cheung KJ, Padmanaban V, Silvestri V, Schipper K, Cohen JD, Fairchild AN, et al. Polyclonal breast cancer metastases arise from collective dissemination of keratin 14-expressing tumor cell clusters. Proc Natl Acad Sci USA. 2016; 113 (7): E854-63. doi: 10.1073/pnas.1508541113.

[9] Szczerba et al. "Neutrophils escort circulating tumr cells to enable cell cycle progression". 2019 Nature, 37-39.

[10] Gkountela S. (2019), Cell 176, 98-112.

[11] Jablonski A., Efficiency of anti-Stokes fluorescence in dyes. In: Nature. Band 131, (1933), S. 839-840.

[12] Cristofanilli M, Budd GT, Ellis MJ, Stopeck A, Matera J, Miller MC, et. al. Circulating tumor cells, diseases progression and survival in metastatic breast cancer NEngl J Med 2004: 351 (8): 781-791.

[13] Bäumler W, Abels C, Karrer S, Weiß T, Messmann H, Landthaler M, Szeimies R Photo-oxidative killing of human colonic cancer cells using indocyanine green and infrared light Br J Cancer (1999) May; 80 (3-4): 360-363.

[14] D. Schikora and M. Weber, British Journal of Cancer, submission\#TH-2019-4280. 